\title{
Estrés percibido, ansiedad, depresión e ira en el proceso de reproducción asistida. Un estudio de caso
}

\author{
Melania Pérez Ortega \\ Instituto de Psicología de Emoción y Salud. Madrid
}

\author{
Jorge Barraca Mairal \\ Universidad Camilo José Cela
}

\section{ResUmen}

En el artículo se presenta un caso donde se analiza la presencia del estrés, la tristeza, la ansiedad, la ira y el desajuste emocional a lo largo de la aplicación de una técnica de reproducción asistida (Microinyección Espermática-ICSI). Se evaluó a una pareja, ambos de 34 años, antes de comenzar ICSI, durante las fases del proceso y posteriormente. Para la evaluación de sintomatología a lo largo de la intervención médica se utilizaron el BDI-II, el STAI, el STAXI-2 y el Cuestionario de Desajuste Emocional y Recursos Adaptativos en infertilidad (DERA). Tal y como se hipotetizó, los resultados evidencian diferencias en las medidas de cada una de las fases de la intervención médica, y también diferencias significativas entre ambos miembros de la pareja, siendo la mujer quien presenta mayores niveles de malestar, exceptuando el momento de la comunicación de resultados (negativos, en este caso), donde la respuesta del marido fue superior.

Palabras clave: Desajuste emocional, estrés, tristeza, ansiedad, ira, infertilidad, técnicas de reproducción asistida, microinyección espermática.

\begin{abstract}
The following review is based on a case research where perceived stress, sadness, anxiety, anger, emotional maladjustment were analysed during the application of an assisted reproduction technique (Intracytoplasmic sperm injection, ICSI). For this procedure a young couple (34 years old) was evaluated in different stages: before ICSI treatment, during the treatment and after it. For symptoms evaluation during the medical intervention, BDI-II, STAI, STAXI-2 and Emotional Maladjustment and Adaptive Resources in infertility questionnaire (DERA) were used as evaluation tools. As expected, final results show many differences in the measurements of each stage and also significant differences between the partners: the woman had higher discomfort levels, except the time of communication of the results (negative in this case), where the man had higher levels than her.
\end{abstract}

Keywords: Emotional maladjustement, stress, sadneess, anxiety, anger, infertility, assited reproducction techniques, Intracytosplasmic sperm injection. 
Son numerosas las evidencias sobre el impacto psicológico de la infertilidad y de los tratamientos asociados a esta (Jenaro, MorenoRosset, Jurado y Robaina, 2008). La infertilidad no puede considerarse en sí misma una psicopatología (Antequera, Moreno-Rosset, Jenaro y Ávila, 2008), pero el proceso que abarca la comunicación del diagnóstico de infertilidad, someterse a Técnicas de Reproducción Asistida (TRA) y los repetidos intentos por conseguir un embarazo, supone un periodo largo y estresante que sitúa a las parejas en riesgo de desarrollar problemas y desajustes emocionales (Jenaro et al., 2008).

De acuerdo con Moreno-Rosset (2010), la infertilidad es la incapacidad para conseguir un embarazo "a término", con un niño nacido sano al finalizar el embarazo, y después de al menos un año de mantener relaciones sexuales habituales y sin utilizar medios anticonceptivos. Por tanto, se refiere a aquellas mujeres que pudiendo concebir, no consiguen culminar un embarazo con el nacimiento. En cambio, cuando no se logra el embarazo, se habla de "esterilidad". Esta se entiende como la imposibilidad absoluta de concebir. No obstante, es cierto que ambos términos suelen utilizarse de forma coloquial como sinónimos.

La OMS incluye a la infertilidad dentro de las enfermedades crónicas, pero posee unos matices que la diferencian del resto de padecimientos físicos. De acuerdo con Llavona (2008), la infertilidad es un problema atípico ya que para la gran mayoría de las personas que la padecen no conlleva ninguna repercusión física negativa. No obstante, la realidad clínica muestra que, a pesar de esto, ante la infertilidad pueden aparecer reacciones emocionales semejantes a las de pacientes con otras patologías, como el cáncer (Antequera, Moreno-Rosset, Jenaro y Ávila, 2008). Otra característica que hace de esta enfermedad un problema tan atípico es que, en la mayoría de los casos, son dos personas las implicadas, independientemente de en cuál de los dos se localice la disfunción, por lo que se extiende a la pareja y por ello se habla de 'parejas infértiles'. Por lo que se puede afirmar, según Moreno-Rosset (2010), que afecta a ambos miembros de la pareja.

Se estima que en España casi un 15\% de las parejas en edad reproductiva tienen problemas de fertilidad y existe cerca de un millón de parejas que demandan asistencia reproductiva. Los datos epidemiológicos de los últimos años reflejan un incremento de los problemas de fertilidad en las parejas, que cabe atribuir a factores fisiológicos, ambientales y sociodemográficos (SEF, 2011).

A pesar de que se han presentado algunos datos de prevalencia, se considera importante destacar la dificultad para ofrecer tasas precisas de prevalencia e incidencia en infertilidad, ya que muchas personas no son conscientes de padecer la enfermedad y no hay unanimidad en cuanto a los tiempos que deben transcurrir para que sea etiquetada como tal. Lo que sí resulta claro es que la capacidad de concebir es menor a medida que avanza la edad de la mujer, por lo que los tiempos para determinar el diagnóstico de infertilidad deberían establecerse más cortos cuanta mayor edad tenga la mujer (Moreno-Rosset, 2010). Teniendo en cuenta este aspecto, parece lógico que el tiempo que acarrea el estudio de fertilidad se con- 
vierta en un factor estresante. Además debe tenerse en cuenta que la realización de estas pruebas diagnósticas puede suponer una situación de tensión para ambos miembros de la pareja. Independiente de la causa, son muchos los autores y estudios que plantean que la infertilidad resulta una crisis importante en la vida de las parejas que la padecen.

Se debe tener en cuenta que el diagnóstico de infertilidad parece no afectar igual a hombres que mujeres. En una revisión realizada por Veiga, Puccio, y Tamburelli (2013), se detectó que, aunque hay algunos estudios que sugieren que la experiencia de infertilidad es igual de estresante para ambos sexos, la mayoría de los trabajos indica que las mujeres presentan mayor nivel de ansiedad y estrés en comparación con los hombres. Estas autoras consideran que la infertilidad impacta de manera diferente según el género: las mujeres, desde un punto de vista social y cultural, tienden a considerar la maternidad un asunto prioritario en sus vidas, apareciendo sentimientos de vacío cuando existen dificultades de concepción. En cambio, los hombres lo relacionan más con aspectos de la virilidad y la potencia sexual. En relación con estas diferencias de género, se destaca el estudio realizado por Holter, Anderheim, Bergh y Möller (2006), en el cual se concluye que los hombres experimentan menor reactividad emocional durante el tratamiento, pero no difieren de las reacciones emocionales de las mujeres cuando se produce un fracaso en el tratamiento.

Una vez se ha informado a la pareja del diagnóstico de infertilidad, se les ofrece, normalmente en la misma sesión, las posibles so- luciones para su caso. Aunque existen diversas variantes, las TRA pueden clasificarse básicamente en dos: Inseminación artificial (IA) y Fecundación in vitro (FIV). Dentro de esta última se encuentra la Microinyección espermática (ICSI), cuyo objetivo es conseguir una intervención más activa sobre el proceso de fecundación. La ICSI consiste en la introducción del espermatozoide directamente en el interior de cada ovocito y, una vez conseguida la fecundación y los embriones se han desarrollado in vitro, se procede a seleccionar los más adecuados para transferirlos al útero.

La espera y comunicación de resultados es la última fase de los tratamientos con TRA y suele durar entre 15 y 17 días, independientemente de la técnica aplicada. En estos días la pareja experimenta miedos, esperanzas, dudas y mucho nerviosismo: saber si se ha conseguido o no el embarazo resulta ansioso y estresante (Moreno-Rosset, 2010). Transcurrido este tiempo, deberá realizarse la prueba de embarazo. Cuando se logra el embarazo la alegría y la felicidad colman a la pareja, junto con la presencia de miedos lógicos hacia el futuro, ya que aún deben pasar nueve meses para que nazca el niño. Por el contrario, cuando el resultado es negativo se produce una gran desmoralización, con sentimientos de tristeza e indefensión (Moreno-Rosset, 2009).

Según Moreno-Rosset (2008), el proceso emocional que supone tanto la infertilidad, como los tratamientos para esta, es tan importante que resulta imprescindible que el psicólogo forme parte del equipo multidisciplinar que interviene en el proceso. Se debe destacar que no se trata de una población clínica y que 
la prevalencia de alteraciones psicológicas se iguala a la de la población general, es decir, alrededor de un $20 \%$ manifiesta haber recibido diagnósticos de cuadros de ansiedad o del estado de ánimo. Sin embargo, es de destacar que alrededor de un $60 \%$ de mujeres que han comenzado los tratamientos de TRA presentan modificaciones en su estado anímico (MorenoRosset, Antequera, Jenaro y Gómez, 2009).

Existe acuerdo entre distintos trabajos, como los de Moreno-Rosset (2010) o los de Verhaak, Smeenk, Evers, Kremer, Kraaimaat y Braat (2007), a la hora de plantear que el estado emocional de la pareja evoluciona y se modifica según avanza y evoluciona el tratamiento. Estos autores explican que hay diversas diferencias en el estado emocional presente a la hora de realizar las pruebas, recibir el diagnóstico y comenzar el tratamiento, al igual que tampoco permanecerá estático el estado emocional de la pareja cuando los tratamientos no hayan tenido éxito o tengan que ser iniciados otra vez. Por todo esto es de vital importancia que los estudios sean longitudinales, evaluando a los pacientes en las distintas fases de los tratamientos. Sin embargo, la mayoría de estudios han realizado la evaluación psicológica en una sola ocasión y en diferentes momentos del proceso de tratamiento médico, por lo que es difícil extraer conclusiones conjuntas de la literatura.

Debido al conjunto de carencias señaladas en la bibliografía -necesidad de estudios Iongitudinales, evaluación de ambos miembros de la pareja, análisis de los posibles problemas psicológicos previos, toma de medidas a lo largo de los distintos momentos de la intervención-, en este trabajo se presenta un estudio en el cual se acompañó a una pareja sometida a una ICSI a lo largo de todo el proceso y durante el cual se evaluaron distintos aspectos psicológicos. Se hipotetizó, en función de los estudios revisados, que se producirán cambios significativos en las variables descritas (ansiedad, depresión, ira, estrés percibido, desajuste emocional y recursos adaptativos) a medida que avanzase el tratamiento médico, que, en el caso de que el tratamiento médico no fuera efectivo (no se produjera la concepción), el malestar psicológico aumentaría tras la comunicación de resultados, y que existirán diferencias en los cambios observados en función del sexo.

\section{Método}

\section{Participantes}

Los participantes de este estudio de caso fueron un hombre y una mujer. Se evaluó a esta pareja en las distintas fases del tratamiento con TRA, en este caso la ICSI. La pareja está formada por N. (hombre) y E. (mujer), ambos tienen 34 años. Tras 6 meses intentando concebir, deciden realizarse pruebas de fertilidad. Acuden a una Unidad de Reproducción privada. A N. le realizan un espermiograma y un cariotipo en agosto del 2013. A E. se le realizan análisis de sangre de junio a agosto del año 2014, múltiples ecografías vaginales y un cariotipo en agosto. Se diagnóstica a la mujer de "Baja reserva ovárica - FSH Alta". No ha habido embarazos anteriores. Ninguno de los miembros de la pareja ha recibido atención psicológica a lo largo de su vida y tampoco durante el proceso de diagnóstico e intervención de fertilidad. Afirman que hasta ahora nunca se les ha ofrecido atención psicológica de ningún 
tipo en el proceso de TRA, aspecto que consideran muy importante, sobre todo si el resultado del tratamiento es negativo.

\section{Procedimiento}

Se contacta con la pareja por primera vez el 20 de enero del 2014. Se les ofrece información sobre el objetivo del estudio, así como aspectos de confidencialidad y la posibilidad de conocer los resultados finales del presente trabajo. También se les indica el número de cuestionarios que deberán realizar, con una estimación del tiempo que les llevará su realización e instrucciones para su correcta aplicación. La pareja accede a realizar este seguimiento y se muestra colaboradora en cada fase de este: Pre-tratamiento, primera y segunda semana de estimulación ovárica, implantación de embriones, espera de resultados, comunicación de resultados, post-tratamiento y un mes después, a modo de seguimiento. En total, la pareja hubo de completar los cuestionarios en ocho ocasiones, lo que realizaron sin demora ni dificultades, dando muestras así de su implicación y su actitud colaborativa en el presente estudio.

E. comienza el tratamiento de ICSI el 27 de enero de 2014, inyectádose la medicación ese mismo día por la noche. A partir de esa fecha, cada día debe acudir para realizar un control y ajustar la medicación que le han mandado para esta primera fase de la ICSI, denominada "Estimulación ovárica". El tratamiento médico persiste hasta el 24 de febrero, día en el que les comunican que el tratamiento no ha funcionado y les ofrecen como opciones "volver a intentarlo con ICSI con pocas probabilidades o la ovodonación", según refiere $\mathrm{E}$.

\section{Instrumentos}

- Ficha general de datos. Además de los datos sociodemográficos, se recabó información relativa al problema de fertilidad y al nivel de estrés percibido.

- Beck Depression Inventory: BDI-II (Beck, Steer y Brown, 1996. Adaptación española por Sanz y Vázquez, 2011). En su última versión, el instrumento para la medida de la depresión de Beck y colaboradores se compone de 21 ítems indicativos de síntomas tales como tristeza, llanto, pérdida de placer, sentimientos de fracaso y de culpa, pensamientos o deseos de suicidio, pesimismo, etc. El formato de los ítems es de tipo Likert con cuatro categorías de respuesta, que se codifican de 0 hasta 3.La fiabilidad del inventario en términos de consistencia interna es buena (alfa de Cronbach 0,76 - 0,95). La validez convergente del BDI-II se evidencia en el valor que alcanzan sus correlaciones con otras escalas que miden depresión (superiores siempre a 0,50).

- Escala de Ansiedad Estado/Rasgo: STAI-E/R. (Spielberger, Gorsuch, y Lushene, 1982. Versión española del State-Trait Anxiety Inventory, STAI, Spielberger, Gorsuch y Lushene, 1970). EI cuestionario evalúa el nivel actual de ansiedad y la predisposición de la persona a responder con estrés. Está formado por 40 ítems, de los cuales la mitad pertenecen a la subescala Estado, formada por frases que describen cómo se siente la persona en ese momento. La otra mitad, a la subescala Rasgo, identifica cómo se siente la persona habitualmente. La puntuación para cada escala puede oscilar de 0 a 30, indicando las puntuaciones más altas mayores niveles de ansiedad. La consistencia interna 
de la prueba es 0,90-0,93 (subescala Estado) y 0,84-0,87 (subescala Rasgo). La fiabilidad testretest: 0,73-0,86 (subescala Rasgo). Respeto a la validez, el STAI muestra correlaciones con otras medidas de ansiedad, como la Escala de Ansiedad Manifiesta de Taylor y la Escala de Ansiedad de Cattell $(0,73-0,85)$.

- Inventario de Expresión de la Ira Estado- Rasgo: STAXI-2 (Miguel-Tobal, Casado, Cano-Vindel y Spielberger, 2001. Versión española de la prueba State-Trait Anger Expresión Inventory, STAXI-2, de Spielberger, 1988, 1991). Este inventario permite obtener una medida del Rasgo de Ira a través de dos subescalas: Temperamento de Ira y Reacción de Ira. Por otro lado, permite obtener el Índice de Expresión de Ira, a través de las subescalas: Expresión Externa e Interna de Ira y Control Externo e Interno de Ira. En cuanto a las propiedades psicométricas de la prueba, diferentes estudios han mostrado una alta correlación test-retest, con un intervalo de 2 meses, tanto en el Rasgo de Ira $(a=0,71)$ como en las escalas de Expresión ( $a=0,59$ y $0,61)$. Igualmente, los resultados encontrados en todas las escalas y subescalas del STAXI-2 indican una buena consistencia interna, con valores que oscilan entre 0,82, en el Rasgo de Ira, y 0,69 y 0,67 en la Expresión de Ira.

- Cuestionario de Desajuste emocional y Recursos adaptativos en infertilidad: DERA (MorenoRosset, Antequera y Jenaro, 2008). Instrumento compuesto por 48 ítems con un formato de respuesta tipo Likert de cinco puntos, que permite conocer el grado en que el sujeto considera falso o verdadero cada uno de los enunciados que se le presentan. Es el primer instrumento creado en España que proporciona una medi- da fiable de la posible presencia o ausencia de desajuste emocional, a la vez que ofrece información sobre los recursos adaptativos, tanto personales como interpersonales en sujetos infértiles. Posee apropiadas cualidades psicométricas: el coeficiente alfa de Cronbach de la escala global es de 0,85 y en cada una de sus escalas presenta una satisfactoria fiabilidad.

En la Tabla 1 se incluyen los distintos momentos en los que la pareja completó los Cuestionarios y la fase del proceso de reproducción asistida en que se encontraba.

\section{Resultados}

Niveles de estrés percibido

En cada ficha general de datos se preguntaba a la pareja, a través de una escala tipo Likert, del 1 al 7, el nivel de estrés percibido durante la semana. Las respuestas indicadas por ambos miembros de la pareja pueden observarse en la Tabla 2 y en la Figura 1.

Los niveles de estrés percibidos de N. (el hombre) son inferiores a los de E. (la mujer) durante la aplicación de la TRA, N. refiere el mayor nivel de estrés percibido tras la comunicación de resultados, volviendo a puntuar en el post-tratamiento y seguimiento igual que en la fase de pre-tratamiento. E. percibe elevados niveles de estrés durante todo el tratamiento, obteniendo la mayor puntuación en la fase de espera de resultados. Tras esta fase refiere niveles de estrés inferiores a los mencionados durante todo el proceso médico.

\section{Niveles de depresión}

En la Tabla 3 y Figura 2 se muestran las puntuaciones obtenidas en la realización del BDI-II, a lo largo de las distintas fases del tratamiento. 
Tabla 1.

Calendarización de fases de tratamiento.

\begin{tabular}{ll}
\hline Fase de Tratamiento & Fechas de realización de cuestionarios \\
\hline Pre-Tratamiento & 22 de enero del 2014 \\
Estimulación ovárica (Primera semana) & 29 de enero del 2014 \\
Estimulación ovárica (Segunda semana) & 03 de febrero del 2014 \\
Implantaciones de embriones & 09 de febrero del 2014 \\
Espera de resultados & 17 de febrero del 2014 \\
Comunicación de resultados & 02 de marzo del 2014 \\
Post-tratamiento & 23 de febrero del 2014 \\
Seguimiento & 27 de abril del 2014 \\
\hline
\end{tabular}

Tabla 2.

Niveles percibidos de estrés en una escala del 1 al 7 por los participantes del estudio.

\begin{tabular}{lll}
\hline \hline Momento de evaluación & N. & E. \\
\hline Pre-tratamiento 22/01 & 1 & 5 \\
Estimulación Ovárica 29/01 & 2 & 6 \\
Estimulación Ovárica 03/02 & 3 & 6 \\
Implantación de embriones 09/02 & 2 & 6 \\
Espera de resultados 17/02 & 3 & 7 \\
Comunicación de resultados 02/03 & 6 & 4 \\
Post-tratamiento 23/03 & 1 & 4 \\
Seguimiento 27/04 & 1 & 4 \\
\hline
\end{tabular}

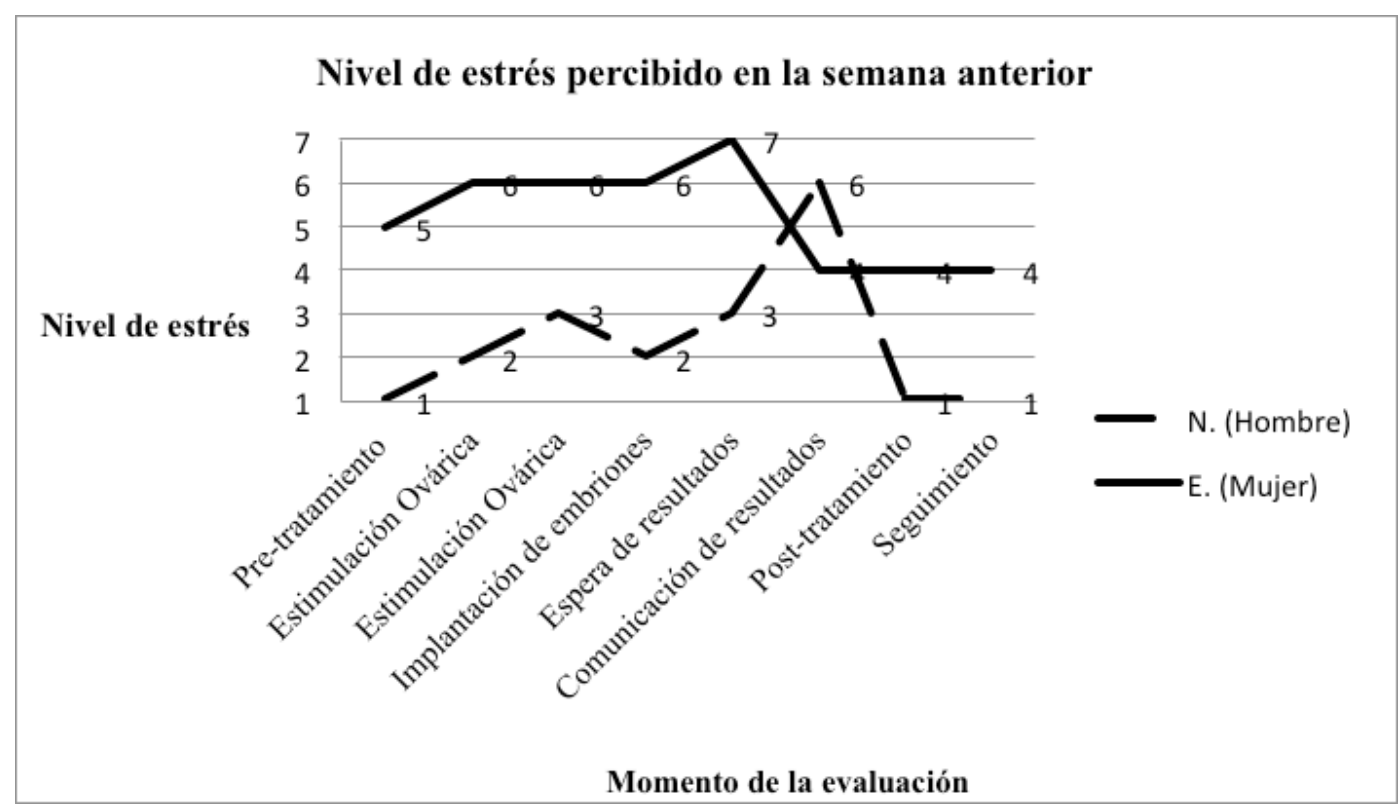

Figura 1. Nivel de estrés percibido por los participantes del estudio. 
En N., hasta la comunicación de resultados, la puntuación refleja: "No depresión (o mínima)". Tras los resultados, la puntuación varía, pasando al rango de puntuación denominado "Depresión severa". Este aumento en sus niveles de tristeza coincide con la información que facilita E. sobre el impacto que tiene la noticia en su marido. Cuando se repite esta prueba a los 20 días, la puntuación ha disminuido, reflejando de nuevo "No depresión (o mínima)."

Las puntuaciones de E. presentan diferencias entre los distintos momentos de evaluación. Antes de comenzar el tratamiento y en la primera semana de tratamiento, la puntuación se encontraría dentro del rango de "Depresión leve". En la segunda semana de tratamiento, la puntuación de E. se encuentra en el rango de "Depresión moderada", que vuelve a alcanzarse en la evaluación que se realiza tras la comunicación de los resultados y en la fase de seguimiento. En el resto de medidas tomadas, es decir tras la implantación de embriones, en la fase de espera de resultados y en la medida post-tratamiento, E. obtiene puntuaciones que se corresponda de nuevo a "Depresión leve".

Tabla 3.

Puntuaciones obtenidas en el BDI-II para los participantes del estudio.

\begin{tabular}{lll}
\hline \hline Momento de evaluación & N. & E. \\
\hline Pre-tratamiento 22/01 & 6 & 14 \\
Estimulación Ovárica 29/01 & 4 & 18 \\
Estimulación Ovárica 03/02 & 3 & 20 \\
Implantación de embriones 09/02 & 7 & 17 \\
Espera de resultados 17/02 & 9 & 19 \\
Comunicación de resultados 02/03 & 31 & 23 \\
Post-tratamiento 23/03 & 12 & 19 \\
Seguimiento 27/04 & 7 & 21 \\
\hline
\end{tabular}

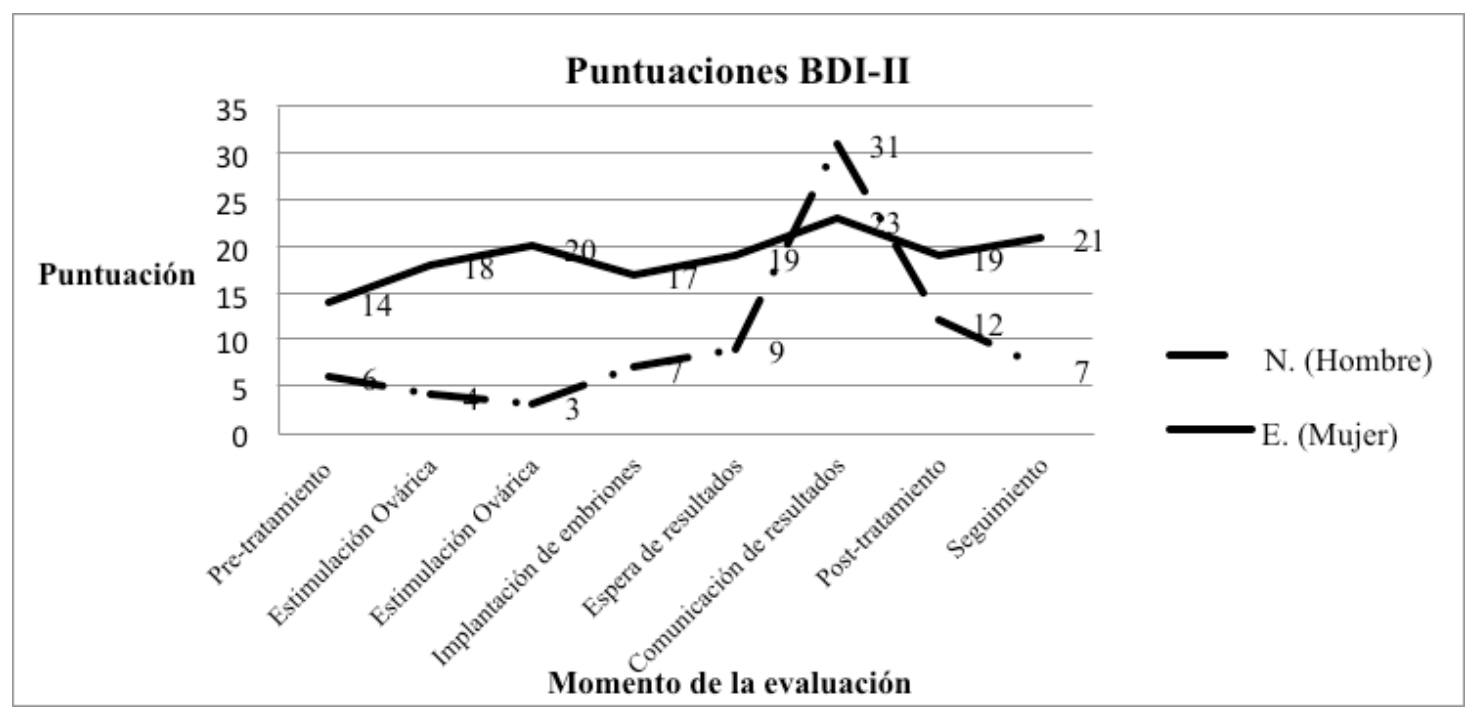

Figura 2. Puntuaciones obtenidas en el BDI -II por los participantes del estudio. 
Las puntuaciones de E. son superiores a las de N. durante todo el proceso, a excepción de la fase de comunicación de resultados, en la cual $\mathrm{N}$. obtiene una puntuación más elevada que $\mathrm{E}$.

Niveles de ansiedad-estado

En la Tabla 4 y Figura 3 se detallan las puntuaciones obtenidos por ambos miembros de la pareja en la escala Estado del STAI.

En N. se observa un leve descenso progresivo en el transcurso de las dos primeras se- manas, para volver a obtener una puntuación idéntica a la del pre-tratamiento tras la fase de implantación. Se produce un alto incremento de la puntuación tras la comunicación de resultados, que comienza a disminuir en el posttratamiento, llegando a niveles más bajos de los iniciales en la fase de seguimiento. E. no muestra diferencias tan marcadas como las que se pueden observar en N. pero también se observa un incremento de su puntuación,

Tabla 4.

Puntuaciones obtenidas en la escala Estado (STAI) por los participantes del estudio.

N.

Momento de evaluación

Pre-tratamiento 22/01

Estimulación Ovárica 29/01

Estimulación Ovárica 03/02

Implantación de embriones 09/02

Espera de resultados 17/02

Comunicación de resultados 02/03

Post-tratamiento 23/03

Seguimiento $27 / 04$

PC

$14 \quad 25$

12

10

14

10

31

22

10
PC

25

20

15

25

15

80

65

15
E. PC 75 85 89 90 85 95 85 89

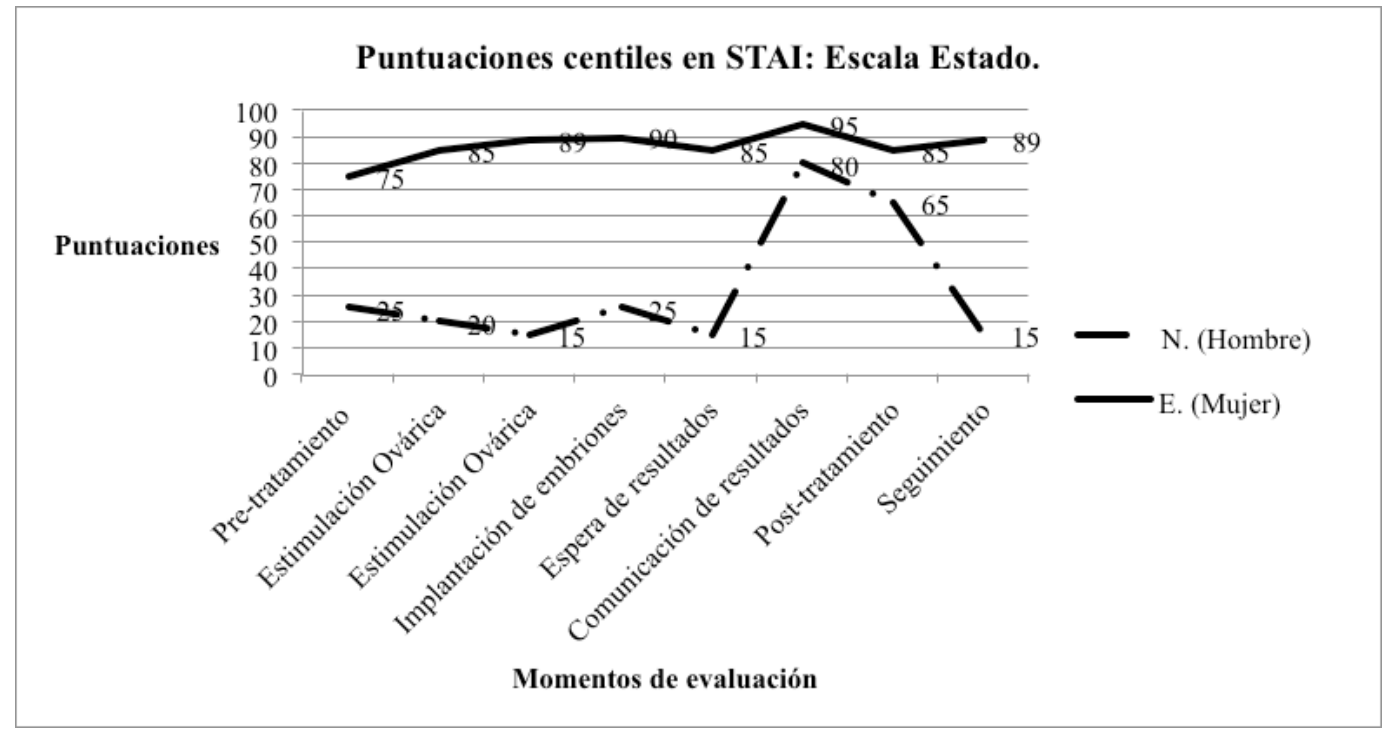

Figura 3. Puntuaciones obtenidas en STAI, escala Estado, por los participantes del estudio. 
siendo la mayor obtenida, tras la comunicación de los resultados.

\section{Niveles de ira}

En la escala Rasgo como se observa en la Figura 4, N. obtiene puntuaciones más elevadas a medida que avanza el tratamiento, exceptuando la fase de espera de resultados donde se da un ligero descenso de acuerdo a la fase anterior de implantación de embriones. Alcanza la máxima puntuación en la fase de comunicación de los resultados, y luego disminuye ligeramente una vez finaliza el proceso. Mientras que E. obtiene en esta escalas puntuaciones elevadas a lo largo de todo el proceso.

En la escala de Expresión Externa, como se observa en la Figura 5, las puntuaciones que obtiene N. no son muy elevadas, exceptuando la puntuación tras la comunicación de resultados, en la cual se produce un gran incremento. Esta se reduce de nuevo en las medida posttratamiento y continúa siendo más baja en la medida realizada en el seguimiento. En el caso de E., se obtienen puntuaciones bajas que se mantienen estables antes y durante la realización del tratamiento. Después de este aumentan, pero sin superar el percentil 40.

Al hablar de la escala de Expresión interna, N. muestra en la mayoría de las fases de evaluación puntaciones que se sitúan entre el percentil 30 y 55, exceptuando las fases espera y comunicación de resultados. En la medida de seguimiento se da un leve incremento en relación con la medida de pos-tratamiento, pasando de un percentil 55 a un percentil 65. En E. se observan puntuaciones elevadas, obteniendo las más altas en la fase de implantación de embriones y de post-tratamiento.

En relación con la escala de Control Externo, puede observarse, en la Figura 7 una gran variedad de puntuaciones obtenidas por N. a lo largo del tratamiento, pasando por niveles elevados, medios y bajos. En el caso de E. no sucede lo mismo, sus puntuaciones son mucho más esta-

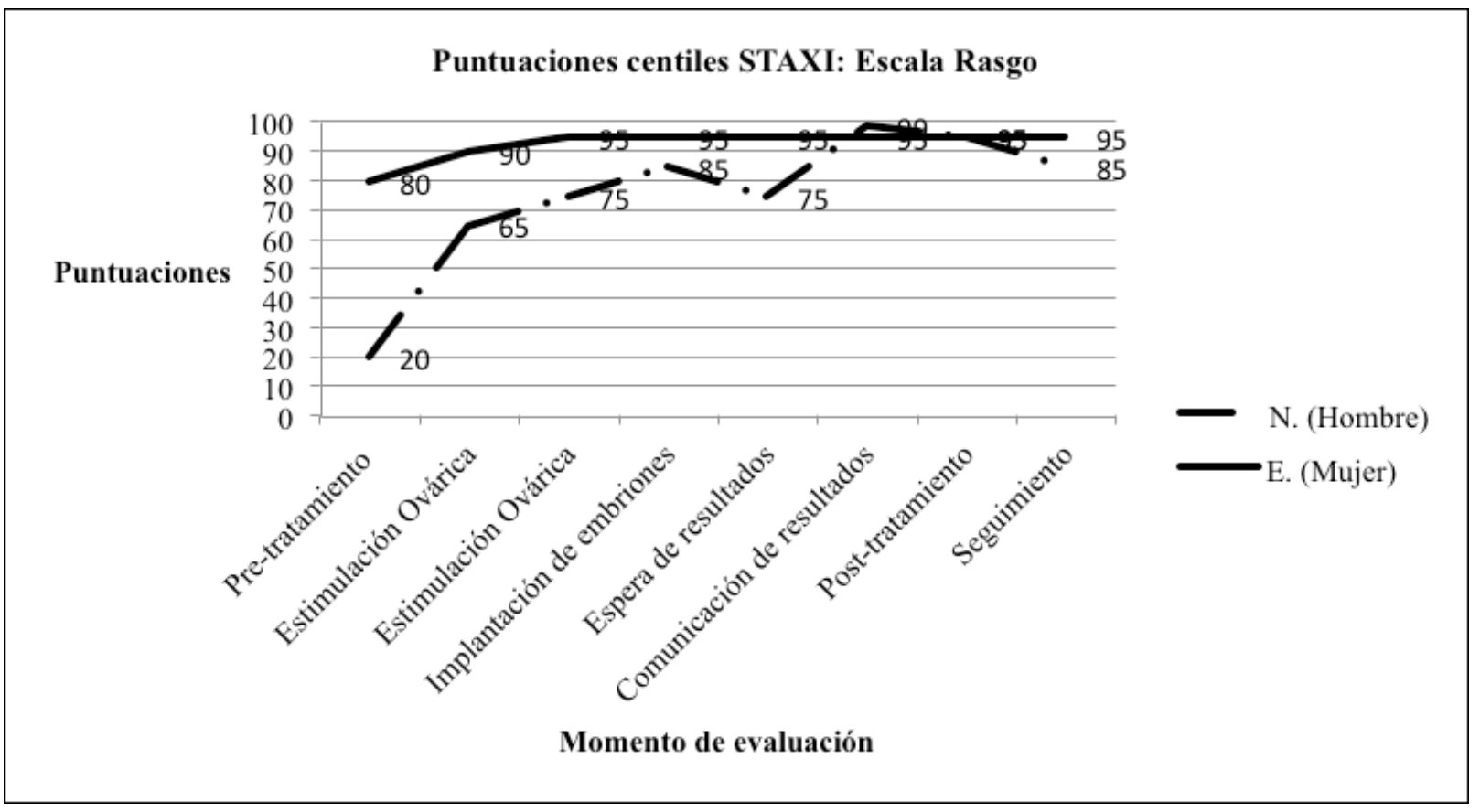

Figura 4. Puntuaciones obtenidas en STAXI, en la escala de Rasgo, por los participantes del estudio. 


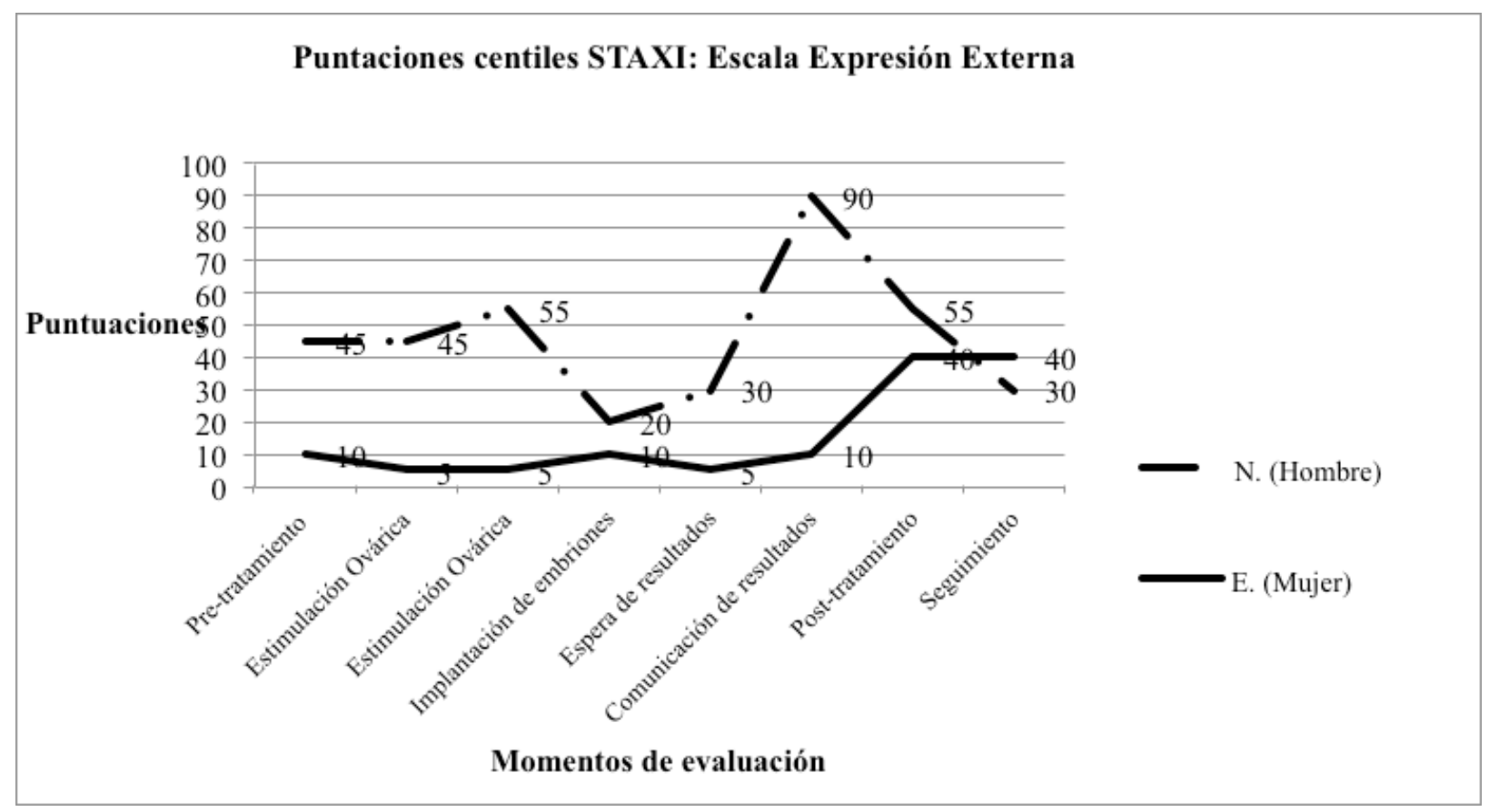

Figura 5. Puntuaciones obtenidas en STAXI, en la escala de Expresión Externa por los participantes del estudio.

bles, manteniéndose elevadas durante todo el proceso. En el Control Interno, como se muestra en la Figura 8, se observa algo similar: puntuaciones muy variadas en $\mathrm{N}$. a lo largo del proceso de evaluación, y elevadas y constantes en $\mathrm{E}$.
Desajuste emocional y recursos adaptativos

En la Tabla 5 se pueden observar las puntuaciones obtenidas en el DERA en los distintos momentos en los que este instrumento fue realizado por ambos miembros de la pareja.

\section{Puntaciones centiles STAXI: Escala Expresión Interna}

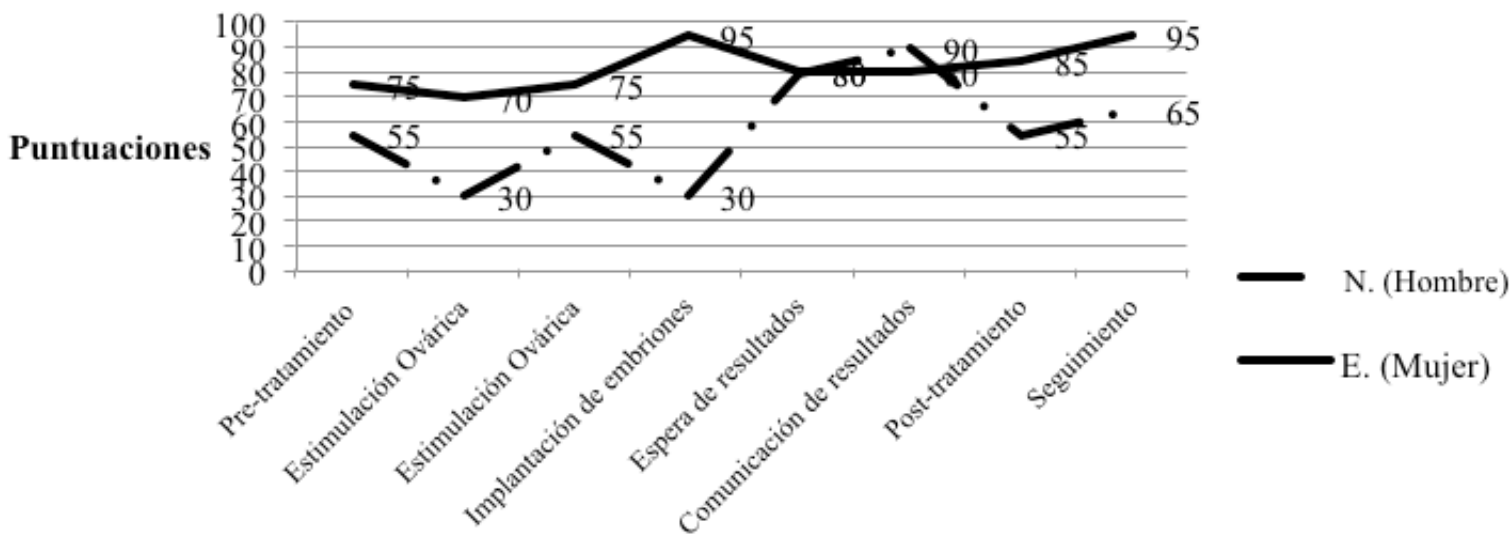

Momentos de evaluación

Figura 6. Puntuaciones obtenidas en STAXI, en la escala de Expresión Interna por los participantes del estudio 


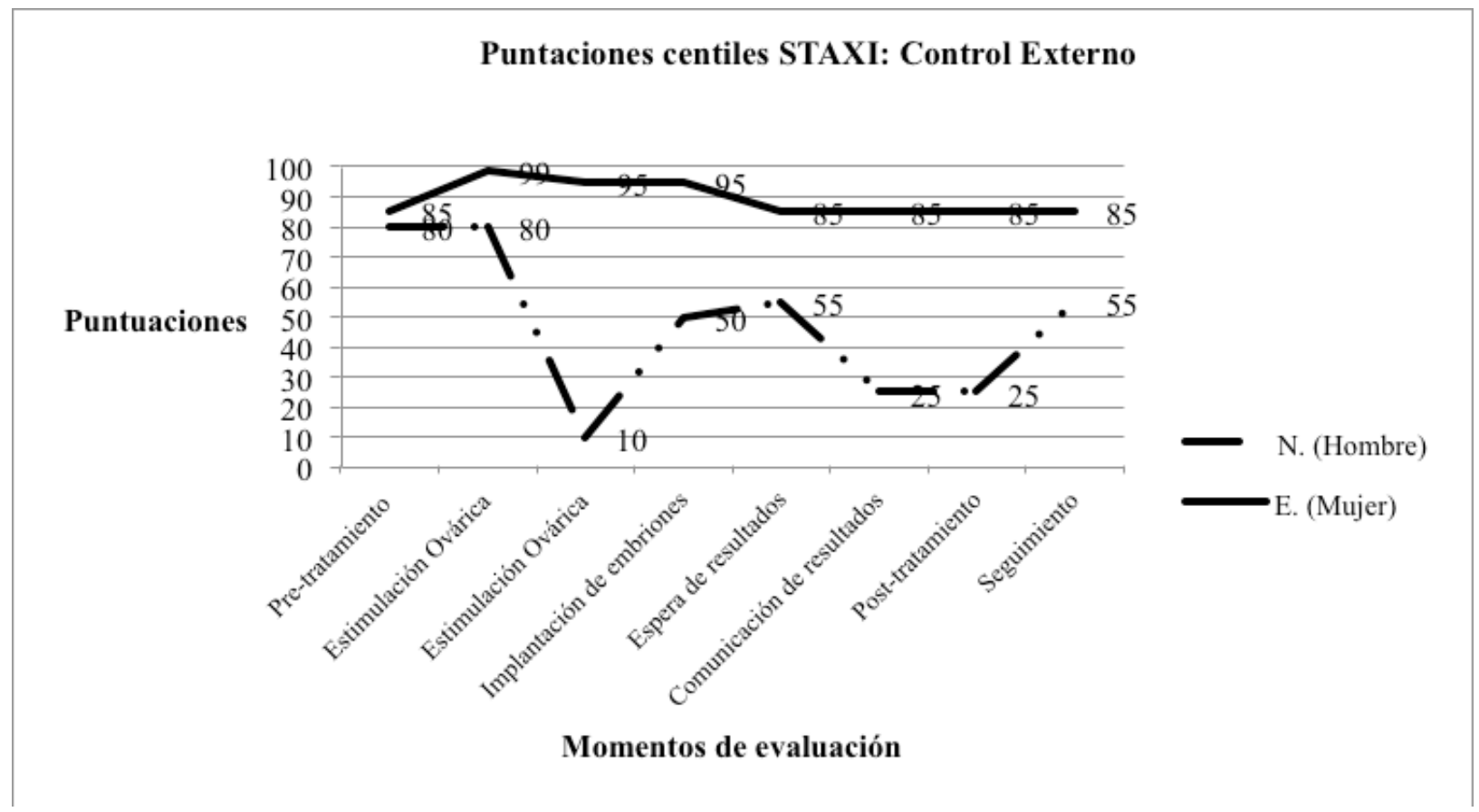

Figura 7. Puntuaciones obtenidas en STAXI, en la escala de Control Externo por los participantes del estudio.

\section{Puntaciones centiles STAXI: Control Interno}

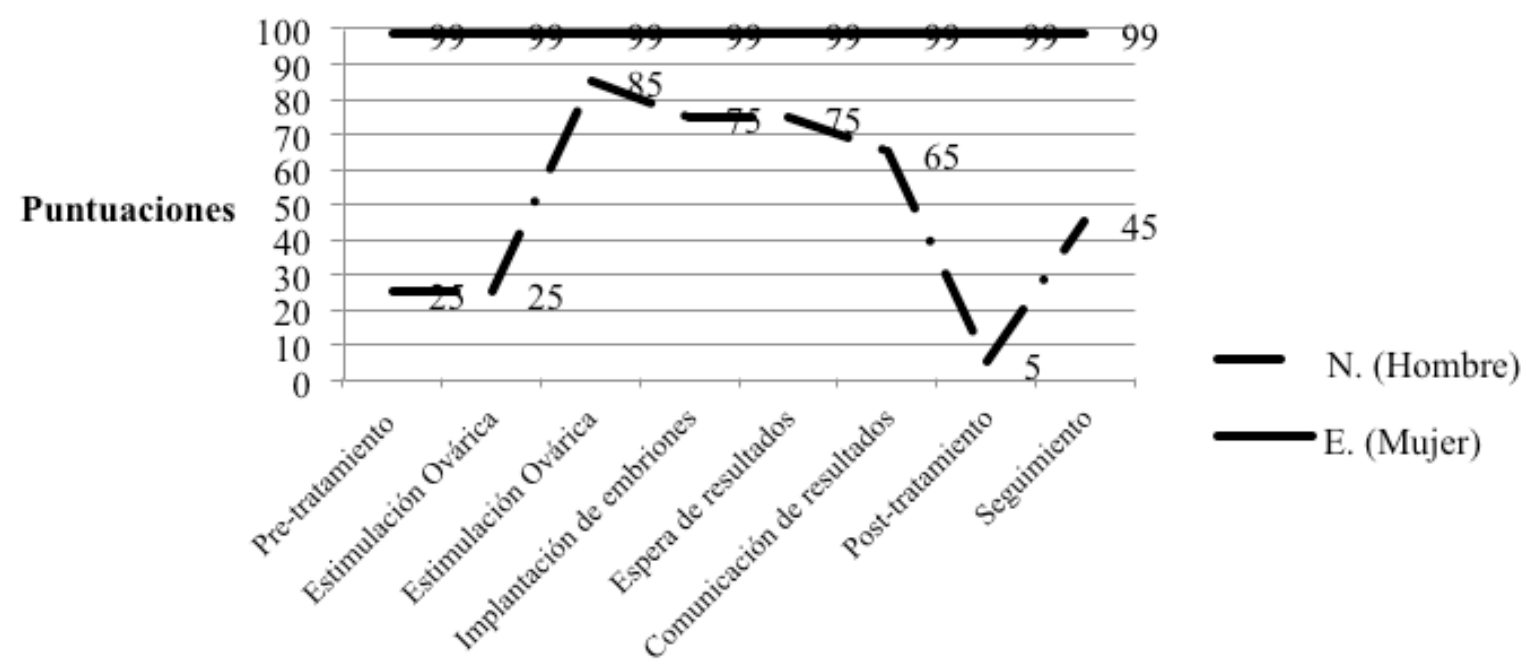

Momentos de evaluación

Figura 8. Puntuaciones obtenidas en STAXI, en la escala de Control Interno por los participantes del estudio.. 
Tabla 5.

Puntuaciones obtenida a través del DERA por los participantes de este estudio.

\begin{tabular}{|c|c|c|c|c|c|c|c|c|c|c|c|c|}
\hline & \multicolumn{4}{|c|}{$\begin{array}{l}\text { Tras la comunicación de } \\
\text { los resultados } 02 / 03\end{array}$} & \multicolumn{4}{|c|}{ Post-tratamiento 23/03 } & \multicolumn{4}{|c|}{ Seguimiento 27/04 } \\
\hline & \multicolumn{2}{|c|}{ N. } & \multicolumn{2}{|c|}{ E. } & \multicolumn{2}{|c|}{ N. } & \multicolumn{2}{|c|}{ E. } & \multicolumn{2}{|c|}{ N. } & \multicolumn{2}{|c|}{ E. } \\
\hline & PD & PC & PD & $\mathrm{PC}$ & PD & $P C$ & PD & $\mathrm{PC}$ & PD & PC & PD & $\mathrm{PC}$ \\
\hline $\begin{array}{l}\text { Desajuste } \\
\text { emocional }\end{array}$ & 46 & 30 & 84 & 90 & 57 & 55 & 85 & 90 & 47 & 30 & 92 & 97 \\
\hline $\begin{array}{l}\text { Recursos } \\
\text { personales }\end{array}$ & 32 & 3 & 43 & 65 & 30 & 2 & 41 & 45 & 24 & 1 & 37 & 15 \\
\hline $\begin{array}{l}\text { Recursos } \\
\text { interpersonales }\end{array}$ & 48 & 85 & 45 & 50 & 43 & 50 & 48 & 75 & 42 & 40 & 46 & 60 \\
\hline $\begin{array}{l}\text { Recursos } \\
\text { adaptativos }\end{array}$ & 80 & 35 & 88 & 60 & 73 & 15 & 89 & 65 & 66 & 4 & 83 & 30 \\
\hline
\end{tabular}

Este cuestionario establece tres rangos de puntuaciones a través de los centiles: Posición baja (percentil 25 o inferior), Posición media (entre percentil 25 y 75) y Posición alta (percentil 75 o superior). A través de las Figuras 9, 10, 11 y 12 se muestra cómo han evolucionado las puntuaciones centiles de ambos miembros de la pareja a lo largo del proceso.
Ninguno de los miembros de la pareja obtiene puntuaciones bajas en la escala de desajuste emocional. Sin embargo, en la primera y última medida, N. se encuentra cerca de este rango, obteniendo una puntuación directa de 46 y 47, lo que equivale a una puntuación centil de 30. Puntuaciones bajas en esta escala indican que la persona se encuentra libre de

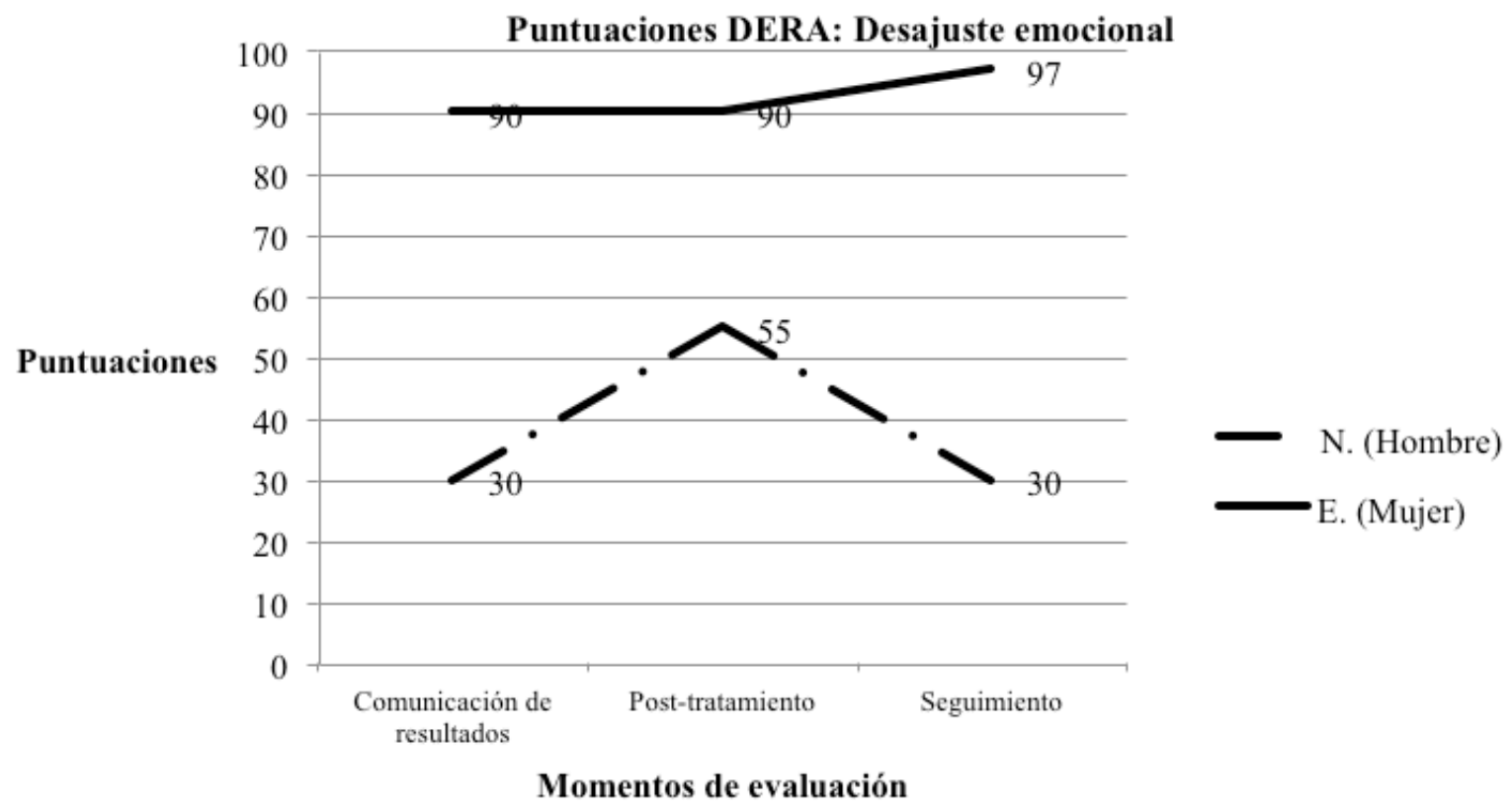

Figura 9. Puntuaciones centiles del DERA: Escala Desajuste emocional, obtenidas por los participantes de este estudio. 


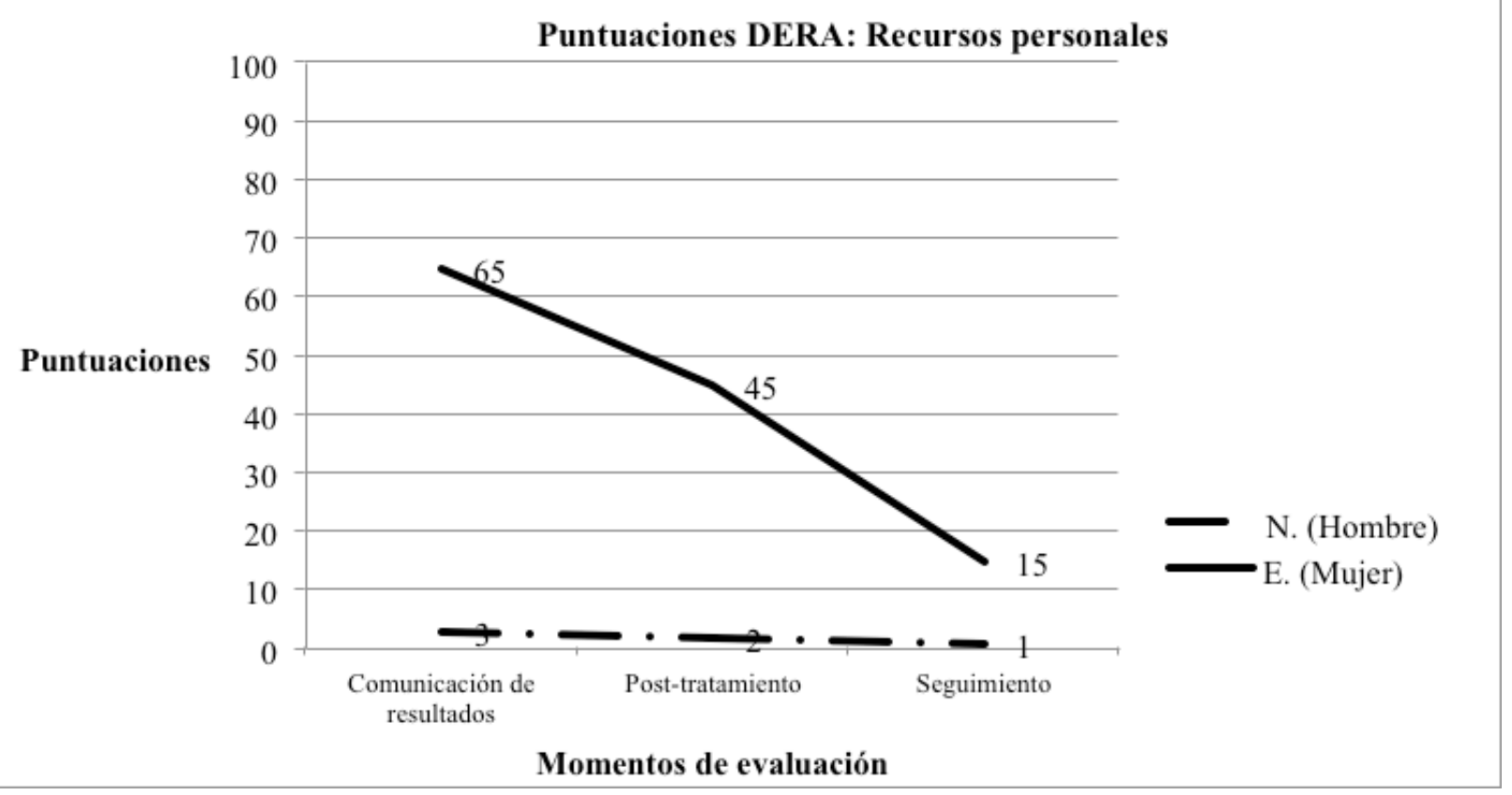

Figura 10. Puntuaciones centiles del DERA: Escala Recursos personales, obtenidas por los participantes de este estudio.

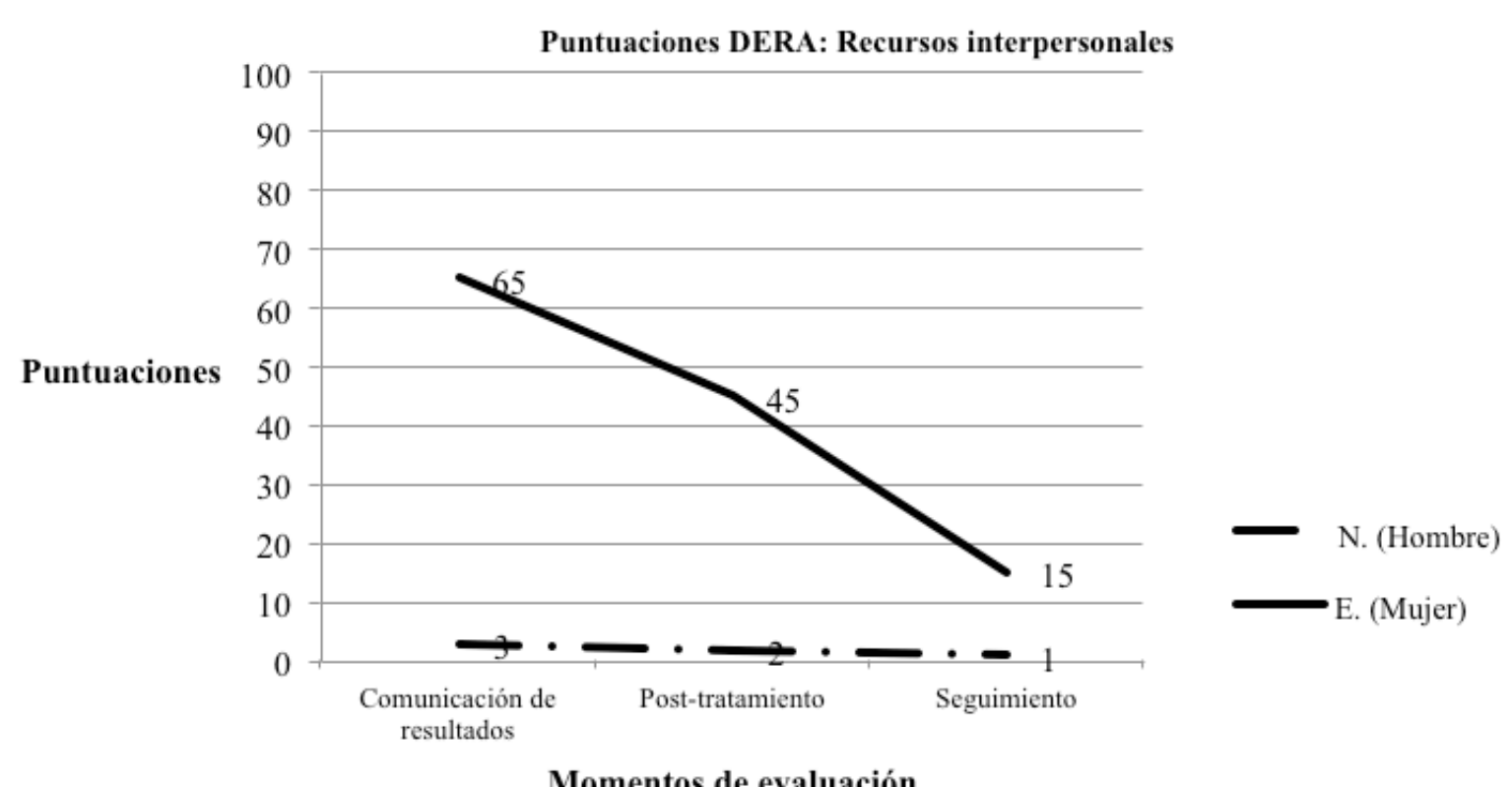

Momentos de evaluación

Figura 11. Puntuaciones centiles del DERA: Escala Recursos interpersonales, obtenidas por los participantes de este estudio. 
procesos psicopatológicos relacionados con la ansiedad o con trastornos del estado de ánimo y que no presenta una especial prevalencia de emociones negativas, de tal manera que en esos momentos de la evaluación, N. es capaz de mantener la estabilidad emocional y preservar su equilibrio personal ante las situaciones en las que se encuentra. No obstante, en el segundo momento de aplicación de este instrumento, las puntuaciones de N. se elevan, pasando a ser considerada su posición como media, obteniendo un centil 55.

Las puntuaciones de E. en esta escala de desajuste emocional se encontrarían en un nivel alto, obteniendo en las dos primeras un percentil 90 y en la última un percentil 97 . Puntuaciones altas en esta escala indican la existencia de emociones que generan un estado de malestar e incrementa su vulnerabilidad a situaciones estresantes, ante las que se puede adoptar una actitud pesimista. Puede aparecer sintomatología cognitiva, motora o fisiológica propia de los estados emociones negativos, así como sentimientos de inseguridad sobre su capacidad para afrontar exitosamente los problemas. Igualmente puede indicar (especialmente cuando las puntuaciones sobrepasan notablemente el percentil 75, como es en el caso de E. en todos los momentos de la evaluación) la posible presencia de problemas de ansiedad o depresión que debieran ser objeto de una evaluación más detallada, con objeto de poder emitir un diagnostico diferencial de trastorno de ansiedad o de trastorno del estado de ánimo.

En la escala de recursos adaptativos, la cual se obtiene a través de la suma de las escalas recursos personales y recursos interpersonales, $\mathrm{N}$. obtiene puntuaciones más bajas que E., comenzando por un percentil 35 en la primera administración de este instrumento, que desciende hasta un percentil 4 en la última

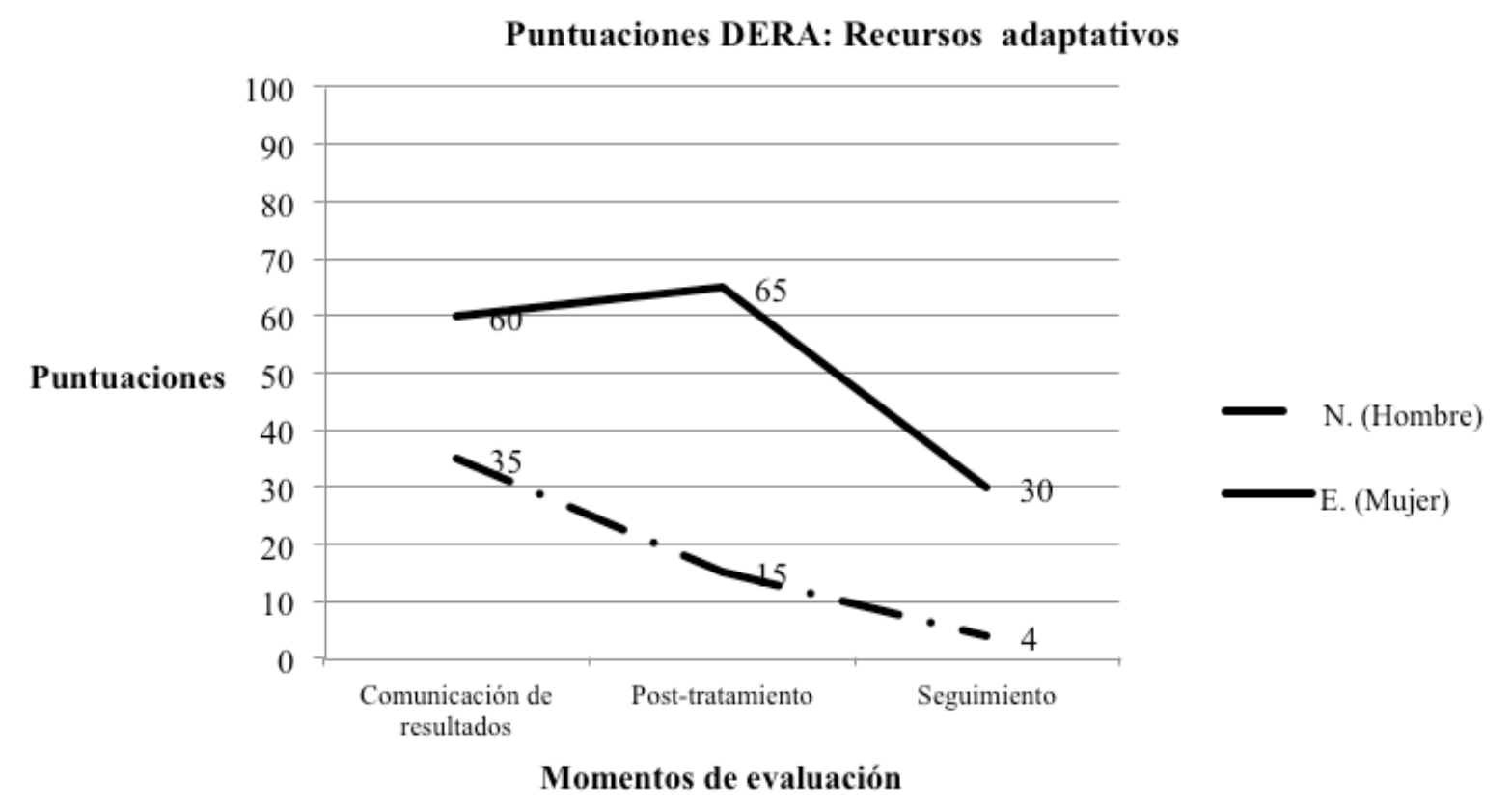

Figura 12. Puntuaciones centiles del DERA: Escala Recursos adaptativos, obtenidas por los participantes de este estudio. 
medida recogida. Puntuaciones bajas en esta escala indican que el sujeto no dispone de estrategias básicas que le permitan afrontar las situaciones estresantes y le ayuden a preservar su equilibrio emocional ante las mismas. N. obtiene muy bajas puntuaciones en la escala de recursos personales, lo que indica una escasa disposición a presentar una actitud activa y de agente de cambio ante los problemas a los que se enfrenta, abandonando la lucha activa y dejándose llevar por los acontecimientos. La interpretación de este instrumento, refleja que N. muestra escasa flexibilidad conceptual en el análisis de las situaciones, sin intentar adoptar distintos puntos de vista ante las mismas o buscando soluciones alternativas. En cuanto a los recursos interpersonales, se puede observar que $\mathrm{N}$. obtiene una puntuación mayor que en los anteriores, obteniendo un percentil 85 en el primer momento de aplicación del instrumento, un percentil 50 en el segundo, y por último una puntuación centil de 40. La puntuación obtenida por N. en el primero momento de evaluación, indica que mantiene una actitud positiva hacia aquellas personas que configuran su medio más cercano (familia, pareja, amigos) haciéndoles partícipes de sus problemas o conflictos personales y sintiendo que estos le pueden ayudar a resolverlo. De igual modo, según el manual de este instrumento, se siente adecuadamente valorado y apreciado por aquellos que le rodean y previsiblemente mantiene una actitud simétrica hacia las necesidades de los demás. Sin embargo, como se ha mencionado anteriormente, esta puntuación desciende hasta un percentil 40 en la fase de seguimiento.
E. se encuentra en el rango de puntuación media en la escala de recursos adaptativos en los distintos momentos de la aplicación del cuestionario. Obtiene puntuaciones similares en los dos primeros momentos de la evaluación, pero se produce una disminución de esta puntuación en la fase de seguimiento. Las puntuaciones en recursos personales descienden desde una posición media en el primer y segundo momento, hasta una posición baja en la fase de seguimiento. En los recursos interpersonales obtiene puntuaciones que varían entre el percentil 50 y 75, obteniendo la máxima puntuación en el segundo momento de evaluación, en la fase de post-tratamiento.

\section{Discusión}

Los resultados obtenidos en la mayoría de las escalas que componen los instrumentos utilizados muestran una gran variedad de puntuaciones, tanto entre los dos miembros de la pareja, como en estos mismos en los distintos momentos de evaluación. Este hallazgo coincide con lo apuntando en los estudios de Antequera et al. (2008) y Verhaak et al. (2007), quienes consideran que el estado emocional de ambos miembros de la pareja no es estáti$\mathrm{co}$, sino que evoluciona y sufre modificaciones a medida que va avanzando el proceso de TRA, existiendo diferencias en el estado emocional presente a la hora de recibir el diagnóstico, comenzar el tratamiento, esperar los resultados y tras la comunicación de estos. A su vez, los resultados aquí obtenidos concuerdan con los del estudio de Moreno-Rosset et al. (2009), en el cual, aunque se incide en la importancia de recordar que las parejas infértiles no se con- 
sideran población clínica, se tiene en cuenta que alrededor de un $60 \%$ de las mujeres que se someten a TRA presentan modificaciones significativas en su estado de ánimo. Por lo tanto, puede considerarse confirmada la hipótesis de la existencia de cambios a lo largo del proceso de tratamiento, considerándose que el momento de aplicación de los instrumento pueden variar las conclusiones de cualquier investigación, y aun más en aquellas realizadas en población con infertilidad, por los matices diferenciales de esta (Moreno-Rosset, 2010).

Se debe destacar que en el caso de E., las puntuaciones reflejan mayores niveles de malestar y permanecen más estables a lo largo del proceso, mientras que en las puntuaciones de N. se pueden observar más diferencias, siendo inferiores a las de su pareja en la mayoría de los momentos de evaluación, como se puede observar en un casi todas las escalas y medidas: niveles de estrés percibido, depresión (BDI-II), ansiedad (STAI), ira (STAXI 2) -escalas de Rasgo, Temperamento, Ira interna, Control externo e interno-y Escala de Desajuste emocional (DERA). Esto supondría la aceptación, en esta pareja, de la hipótesis sobre la existencia de diferencias en función del sexo, lo que concuerda con estudios como el de Veiga et al. (2013) y de Castillo, Moreno-Rosset, Martín y RamírezUclés (2009). En este último se hace referencia a que los niveles de desajuste son superiores en las mujeres, lo que coincide con los resultados obtenidos en este estudio.

A través del DERA se puede observar un descenso de las puntuaciones que hacen referencia a los recursos (personales, interpersonales y adaptativos) de ambos miembros de la pare- ja a medida que avanza el tratamiento $y$, sobre todo, en la fase de seguimiento, aspecto que sería importante comprobar de nuevo pasado un tiempo, con el objetivo de ver si desarrollan estrategias que les permitan aumentar sus recursos sin realizar ninguna intervención psicológica. Por otro lado, también se encuentran coincidencias con el estudio Peterson, Newton y Feingold (2007), de acuerdo con el cual las mujeres experimentan mayores niveles de tristeza y ansiedad durante el proceso que conllevan las TRA, como puede observarse en los resultados que hacen referencia a los niveles de tristeza y ansiedad de este estudio. Se debe destacar en la puntuaciones de N. se observa un claro aumento en los niveles de estrés, tristeza, ansiedad e ira (escala rasgo y expresión, mientras que no se refleja aumento en las puntuaciones de control) tras la comunicación de resultados, incluso superando en varias ocasiones las puntuaciones obtenidas por E. Estos resultados se asemejan a los obtenidos en el estudio de Holter et al. (2006), que concluía que los hombres experimentan menores niveles en cuanto a la reacción emocional durante el tratamiento, pero que su reacción emocional no difería de la de la mujer cuando se produce un fracaso en la fecundación. Estas diferencias en función del sexo deberían tenerse en cuenta a la hora de establecer protocolos de intervención para estas parejas. Como proponen del Castillo et al. (2009), sería recomendable seguir investigando con el objetivo de poder diseñar protocolos de intervención psicológica no sólo específicos para el momento del tratamiento, sino también adaptados al género de la persona a la que va dirigido, puesto que 
los correlatos psicológicos de ambos miembros de la pareja pueden presentarse de forma diferente (del Castillo, Moreno-Rosset, Martín, García-Fernández y Urries, 2008).

Dado los hallazgos de este trabajo, se destaca la importancia de que futuros estudios sigan una metodología longitudinal, puesto que el estado anímico de estas parejas es algo dinámico, que evoluciona a lo largo del proceso de tratamiento, y que se tenga en cuenta de cara a la evaluación a ambos miembros de la pareja.

Como limitaciones de este estudio debe mencionarse, en primer lugar, que no se obtuvo una medida en el momento inicial, cuando la pareja recibió el diagnóstico de infertilidad. La obtención de esta medida hubiera sido muy útil para contrastar adecuadamente la evolución del estado anímico de la pareja.

Otras limitaciones de este trabajo son comunes al resto de estudios con población infértil. La primera sería que las muestras están formadas por parejas que aceptan someterse a técnicas de reproducción, por lo que los resultados no son generalizables a aquellas parejas que optan por no hacerlo. La segunda hace referencia al uso de medidas repetidas que puede originar que los sujetos recuerden lo contestado en otras ocasiones y que esto pueda dar lugar al mismo tipo de respuestas. Por último destacar que lo idóneo hubiera sido realizar este estudio con más parejas, adecuados análisis estadísticos e intentando solventar estas limitaciones para poder obtener resultados generalizables.

Otro aspecto con lo que debería trabajarse, de acuerdo a lo planteado recientemente por
Boden (2013), es lo que sucede ya finalizado el tratamiento, una vez que la pareja decide no intentarlo más. Es lo que este autor define como "el final de la esperanza". No es sencillo renunciar a la esperanza de tener hijos, se tiende a pensar en la evolución de las TRA y en la posibilidad de una concepción espontánea. Pero son conocidos los casos en los que la pareja termina consiguiendo el hijo deseado de una forma natural una vez abandonado el tratamiento. Desgraciadamente esto no es lo más común y supone una posibilidad de esperanza para las parejas, esperanza que, en ocasiones, puede resultar contraproducente para superar adecuadamente este impacto vital tan estresante. Por ello debe comenzar a plantearse, como afirma este autor, como uno de los objetivos de la Psicología de la Reproducción, más allá de lo que sucede en las TRA, ya que el carácter de estas es temporal. Esto es especialmente importante en los casos donde se da infertilidad de tipo idiopático.

Por último, se considera que una línea interesante para futuros trabajos tiene que ver con el peso de las emociones positivas en este proceso y plantear investigaciones longitudinales que evalúen también estos aspectos.

\section{Referencias}

Antequera, R., Moreno-Rosset, C., Jenaro, C. y Ávila, A. (2008). Principales trastornos psicológicos asociados a la infertilidad. Papeles del Psicólogo, 29 (2), 167-175.

Beck, A., Steer, R. y Brown, G. (1996). BDI-II. Beck Depression Inventory-Second Edition. Manual. San Antonio, TX: The Psychological Corporation.

Boden, J. (2013). The ending of treatment: the ending of hope? Human fertility: journal of the British Fertility Society, 16 (1), 22-25. 
Del Castillo, M., Moreno-Rosset, C., Martín, M.D. y Ramírez-Uclés, I. (2009). Diferencias de género en afecto, desajuste emocional y recursos adaptativos en parejas infértiles: Un enfoque positivo. Anuario de Psicología Clínica y de la Salud, (5), 41-48.

Del Castillo, M., Moreno-Rosset, C., Martín, M.D., García-Fernández, E.y Urries, A. (2008). Evaluación de afectos y emociones en parejas infértiles. Comunicación presentada al VII Congreso Internacional de la Sociedad Española para el estudio de la ansiedad y el estrés (SEAS). Benidorm (Alicante).

Holter, H., Anderheim, L., Bergh, C. y Möller, A. (2006). First IVF treatment-short- term impact on psychological well-being and the marital relationship. Human Reproduction 12 (21), 3295-3302.

Jenaro, C., Moreno-Rosset, C., Antequera, R. y Robaina, N. (2008). La evaluación psicológica en infertilidad: el" DERA" una prueba creada en España. Papeles del psicólogo, 29 (2), 176-185.

Llavona, L.M. (2008). El impacto psicológico de la infertilidad. Papeles del Psicólogo, 29 (2), 158-166

Miguel-Tobal, J. J., Casado, M. I., Cano-Vindel, A. y Spielberger, C. D. (2001). Staxi-2: Inventario de expresión de la ira estado rasgo. Madrid: TEA Ediciones, S.A.

Moreno-Rosset C. (2010). Infertilidad. Madrid: Síntesis.

Moreno-Rosset, C. (Coord.) (2009). Infertilidady reproducción asistida. Guía práctica de intervención psicológica. Madrid: Pirámide.

Moreno-Rosset, C. (2008). Infertilidad y psicología de la reproducción. Papeles del Psicólogo, 29 (2), 154-157.

Moreno-Rosset, C., Antequera, R., Jenaro, C., y Gómez, Y. (2009). La psicología de la reproducción: la necesidad del psicólogo en las unidades de reproducción humana. Cínica y Salud, 20 (1), 79-90

Moreno-Rosset, C., Antequera, R. y Jenaro, C. (2008). DERA. Cuestionario de Desajuste emocional y Recursos adaptativos en infertilidad. Madrid: TEA Ediciones, S.A.

Peterson, B. D., Newton, C. R. y Feingold, T. (2007). Anxiety and sexual stress in en and women undergoing infertility treatment. Fertility and sterility, 88 (4), 911914.

Sociedad Española de Fertilidad (2011). La Infertilidad en España: Situación Actual y Perspectivas. Barcelona: Grupo Grafico.

Spielberger C.D., Gorsuch, R.L., y Lushene, R.E. (1982). Cuestionario de Ansiedad Estado/ Rasgo. Madrid:TEA Ediciones

Veiga, M.C., Puccio, M.C. y Tamburelli, V. (2013). ¿Qué relación existe entre estrés, a n s i e dad e infertilidad? Reproducción, 28 (3), 7989.

Verhaak, C., Smeenk, J., Evers, A., Kremer, J., Kraaimaat, F. y Braat, D. (2007). Women's emotional adjustment to IVF: a systematic review of 25 years of research. Human reproduction update, 13 (1), 27-36. 
